\title{
Modelling the mechanical action of a front loading washing machine
}

\author{
Melanie E. Roberts ${ }^{1} \quad$ Celia Kueh $^{2} \quad$ Emma Greenbank ${ }^{3}$ \\ Daniel Clarke ${ }^{4} \quad$ Sibylle van Hove ${ }^{5} \quad$ David J.W. Simpson ${ }^{6}$ \\ Alan Williams ${ }^{7} \quad$ James Williams ${ }^{8}$
}

(Received 15 August 2018; revised 30 July 2019)

\begin{abstract}
Fisher \& Paykel Appliances is a major New Zealand appliance manufacturing company who, amongst other products, develops frontloading washing machines. Their development teams are committed to improving the machine performance. Machine performance is a combination of wash performance (soil removal and wear and tear), power and water consumption, and ease of use. The problem Fisher \& Paykel Appliances posed to Mathematics-in-Industry New Zealand was to investigate mathematical models for the wash performance of front-loading washing machines due to mechanical action. Analysis of experimental data supplied by Fisher \& Paykel Appliances did not identify the expected proportional relationship between soil removal
\end{abstract}

DoI:10.21914/anziamj.v59i0.13473, (C) Austral. Mathematical Soc. 2019. Published 2019-08-16, as part of the Proceedings of the 2017 Mathematics and Statistics in Industry NZ Study Group. ISSN 1445-8810. (Print two pages per sheet of paper.) Copies of this article must not be made otherwise available on the internet; instead link directly to the DOI for this article. 
and wear and tear, indicating that both metrics could be simultaneously improved. The experimental data is further used to identify a relationship between drum speed and the spin dynamics, indicating that a re-configuration of the machine vanes could improve wash performance. Physical and numerical models of wash behaviour are developed to explore the relationship between drum speed and the actions of clothing. A video analysis approach demonstrates that a simple video set-up can be used to gain further insight into the trajectories and mixing of both experimental and genuine wash behaviour.

\section{Contents}

1 Introduction and Problem Description

2 Physical Model

M33

2.1 A point mass model . . . . . . . . . . . . . . M34

2.2 The effect of drum speed . . . . . . . . . . . . . . M37

2.3 Incorporating load size . . . . . . . . . . . . . M40

3 Numerical model of Discrete Elements

M41

4 Data Analysis

M46

5 Empirical Clothing Motion

M55

6 Summary

M58

7 Acknowledgements 


\section{Introduction and Problem Description}

Fisher \& Paykel Appliances is a major New Zealand appliance manufacturing company who, amongst other products, develops front-loading washing machines. Their research and development teams are committed to improving washing machine performance, focussing on the consumer experience in addition to standardised testing. Machine performance is a combination of the wash performance, water and power consumption, and ease of use for the customer. From a consumer perspective, the key criteria for wash performance are the degree to which clothes are cleaned, the soil removal, and the degree of wear and tear on clothing due to washing action.

The clothes washing process removes dirt and grease-like products through a combination of chemical, thermal and mechanical action. To a degree, these processes can compensate for each other: for example, additional detergent can be used to compensate for cooler washing temperatures or a shorter wash cycle. The challenge posed to Mathematics-in-Industry New Zealand was to investigate mathematical models for the wash performance of front-loading washing machines due to mechanical action. The mechanical actions for soil removal are garment-to-garment rubbing, within-garment rubbing, and garment-to-washing machine drum-skin rubbing. These actions are influenced by how clothes move around within the washing machine, and thus Fisher \& Paykel Appliances can modify a number of parameters of front-loading machines and wash cycles to change the wash performance. Parameters able to be altered include: drum dimensions, drum skin profile and drum ends, vane placement and geometry, door protrusion, drum rotational speed, rotation schedule, wash time, and water volume. Other factors that influence wash performance due to mechanical action, within the consumer's control, include the volume of clothes washed, mix and type of clothing washed (e.g., towels versus delicates), how the machine is loaded, and the wash program used. By fixing the water temperature, detergent used, and loading factors (load type, loading method, etc.), Fisher \& Paykel Appliances is able to investigate the effect of varying parameters on the wash performance. 
Wash performance testing is performed under controlled conditions; wash temperature, detergent concentration, load composition, load size, soil type and wash program are held fixed to investigate the impact of varying specific machine parameters. Wash performance is measured using two criteria: soil removal (SR) and gentleness of action (GA). To measure wash performance, swatches of special fabrics are attached to garments to provide estimates of soil removal and gentleness of action (or the wear on the swatch). The SR and GA are scored using quantitative measures on a scale of zero to one. For SR, scores closer to one are desirable as this corresponds to a high rate of soil removal, whereas for GA low scores are preferred, as a score of zero corresponds to no wear on the garment. The evenness of the wash is also a performance measure, with a low standard deviation in the SR and GA scores across swatches in a load being desirable.

For the workshop Fisher \& Paykel Appliances made available results from wash performance testing with a prototype machine to inform the investigations. The team focussed on three approaches to understand the impact of machine parameters on the wash performance: data analysis, physical modelling, and image analytics. These approaches are summarised in the sections below.

Fisher \& Paykel Appliances have a long successful association with industry study groups in Australia and New Zealand. Previous studies include a 2005 investigation into temperature control in wash water (Marsh and Wilkins 2005), understanding washing machine dynamics in top-loading machines (Marsh et al. 2005), and more recently a focus on dryers (Fullard et al. 2016).

\section{Physical Model}

The relationship between the washing machine parameters and the dynamics of a wash are explored through two physical models. A simple point mass model of a single clothing item was used to obtain a relationship between the machine drum radius, rotation speed and the motion of the clothing 
item. The second model, based on geometric considerations, incorporates the amount of clothing in the wash and allows the rotation speed at which washing effectiveness is maximised to be determined. To simplify the analysis, the effect of water on clothing motion is neglected in both models.

\section{$2.1 \quad$ A point mass model}

Consider the action of clothing in a front loading washing machine. The clothing will be picked up by vanes fixed to the side of the drum as the drum rotates. In an inertial frame of reference the sum of the gravitational force and normal force, exerted by the drum surface onto the clothes, equals the centripetal force that keeps the clothes in motion. At low spin speeds, when the normal force from the drum tends to zero, the clothing is carried around the side of the drum before falling to the base of the drum to be picked up by a subsequent vane. The point on the drum where the clothing leaves the side of the drum defines the departure angle, while the point of the drum where the clothing lands defines the landing angle.

By approximating a single item of clothing as a point mass on the perimeter of the drum the departure angle, landing angle and landing speed of the item is expressed as a function of the rotational speed and radius of the drum. Figure 1 shows the forces acting on an item of clothing.

Consider a circular drum of radius $r[\mathrm{~m}]$ rotating anticlockwise with constant angular velocity $\omega=\pi \Omega / 30[\mathrm{rad} / \mathrm{s}]$, where $\Omega$ is the rotational speed of the drum [rpm]. The force due to gravity acting on a point with mass $m[\mathrm{~kg}]$, is

$$
\mathrm{F}_{\text {gravity }}=(0,-\mathrm{mg}) \text {, }
$$

where $\mathrm{g}=9.81\left[\mathrm{~m} \mathrm{~s}^{-2}\right]$ is the acceleration due to gravity. While the clothing is attached to the perimeter of the drum, its location is $(r \sin (\omega t),-r \cos (\omega t))$, and thus the centripetal force is

$$
F_{\text {centripetal }}=\left(-m \omega^{2} r \sin \theta, m \omega^{2} r \cos \theta\right),
$$


Figure 1: Schematic of the motion of a point mass clothing item within the front loader washing machine drum.

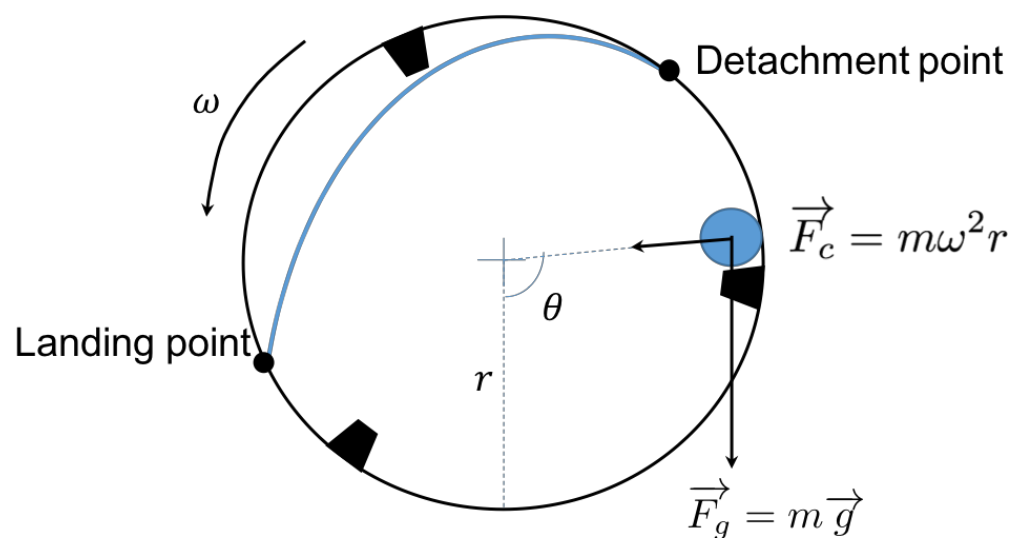

where $\theta=\omega t$ is the angle denoting the position on the drum perimeter of the point mass. In two-dimensional cartesian coordinates with the origin at the centre of the drum the angle $\theta$ is defined anticlockwise from the negative $y$ axis as done by Yun and Park (2015) and Lee et al. (2007). This choice for the orientation of $\theta$ was selected as the base of the drum corresponds to the natural rest point for clothes within the drum. Consequently, observations typically reference this location. We therefore choose to use this mathematically unconventional orientation such that the results are more intuitive, especially since the drum will rotate in both directions at different times during a standard machine cycle. Equation (2) assumes that $\boldsymbol{t}=\mathbf{0}$ corresponds to the point mass being at the base of the drum. Friction between the drum surface and the item of clothing is assumed to be negligible.

The clothing detaches from the side of the drum when the radial component of the gravitational force, $-\mathbf{m} \mathbf{g} \cos \theta$, equals the centripetal force (2). This 
occurs when

$$
\cos \theta_{\text {crit }}=-\frac{\omega^{2} r}{g} .
$$

Thus the departure angle $\theta_{\mathrm{d}}=\theta_{\text {crit }}$.

Once the item of clothing has detached from the inside of the drum, its motion is determined by assuming the kinematic motion of a projectile. This assumption is appropriate as air resistance is negligible and therefore gravity is the only force acting on the mass. Assuming free-fall, the trajectory of the mass from departure to its subsequent landing position in the drum is determined.

When the point mass detaches from the drum perimeter we assume it to undergo free-fall until colliding with the drum. While in flight the position of the point mass is

$$
\begin{aligned}
(x(t), y(t))=( & r \sin \left(\theta_{d}\right)+\omega r \cos \left(\theta_{d}\right) t, \\
& \left.-r \cos \left(\theta_{d}\right)+\omega r \sin \left(\theta_{d}\right) t-\frac{g t^{2}}{2}\right),
\end{aligned}
$$

where $t=0$ is the time the mass detaches from the drum.

The point mass hits the drum when $x(t)^{2}+y(t)^{2}=r^{2}$. This equation is quartic in $t$, but $t=0$ is a double root and so by using (3) to solve for $t$ we obtain simply

$$
\mathrm{t}_{\text {flight }}=\frac{4 \omega \mathrm{r} \sin \left(\theta_{\mathrm{d}}\right)}{\mathrm{g}},
$$

for the flight time. Then by solving $y\left(t_{\text {flight }}\right)=-r \cos (\theta)$ for $\theta$, we obtain

$$
\theta_{l}=\arccos \left(\frac{3 \omega^{2} r}{g}-\frac{4 \omega^{6} r^{3}}{g^{3}}\right)
$$

for the angle at which the mass reattaches to the drum. The mass undergoes acceleration due to gravity in the vertical component, while maintaining its 
departure velocity in the horizontal component. Therefore on landing the velocity of the mass is

$$
v_{\text {end of flight }}=\left(\omega r \cos \left(\theta_{\mathrm{d}}\right), \omega r \sin \left(\theta_{\mathrm{d}}\right)-g \mathrm{t}_{\text {flight }}\right) .
$$

Upon hitting the drum, we assume the velocity of the point mass changes from its final velocity at the end of flight, (7), to the velocity of the drum. This implies that upon collision the point mass attaches to the drum and therefore the velocity changes from (7) to

$$
v_{\text {re-attached }}=\left(\omega r \cos \left(\theta_{l}\right), \omega r \sin \left(\theta_{l}\right)\right)
$$

in a very short interval of time.

We assume that the magnitude of the force at reattachment is proportional to $\|\Delta v\|$ where

$$
\Delta v=v_{\text {re-attached }}-v_{\text {end of flight }} .
$$

After the collision the point mass rotates with the drum from $\theta=\theta_{\mathrm{l}}$ to $\theta=\theta_{\mathrm{d}}$ in the time

$$
\mathrm{t}_{\mathrm{drum}}=\frac{\theta_{\mathrm{d}}-\theta_{\mathrm{l}}}{\omega} .
$$

The overall motion of the point mass is periodic with period $T=t_{\text {flight }}+t_{\text {drum }}$. We suggest that the effectiveness of the washing machine over a fixed length of time is proportional to $\|\Delta v\|$ times the number of impacts. This is proportional to $\mathrm{E}=\|\Delta v\| / \mathrm{T}$.

\subsection{The effect of drum speed}

The effect of drum speed and radius on (4) is shown in Figure 2. The trajectories shown are for typical front loader washing machine drum speeds of $40 \mathrm{rpm}, 45 \mathrm{rpm}$ and $50 \mathrm{rpm}$ and for drum radii of $0.2 \mathrm{~m}, 0.25 \mathrm{~m}$ and $0.3 \mathrm{~m}$. 
Figure 2: Departure position and motion of point mass clothing item with variations of drum speed and drum radius.

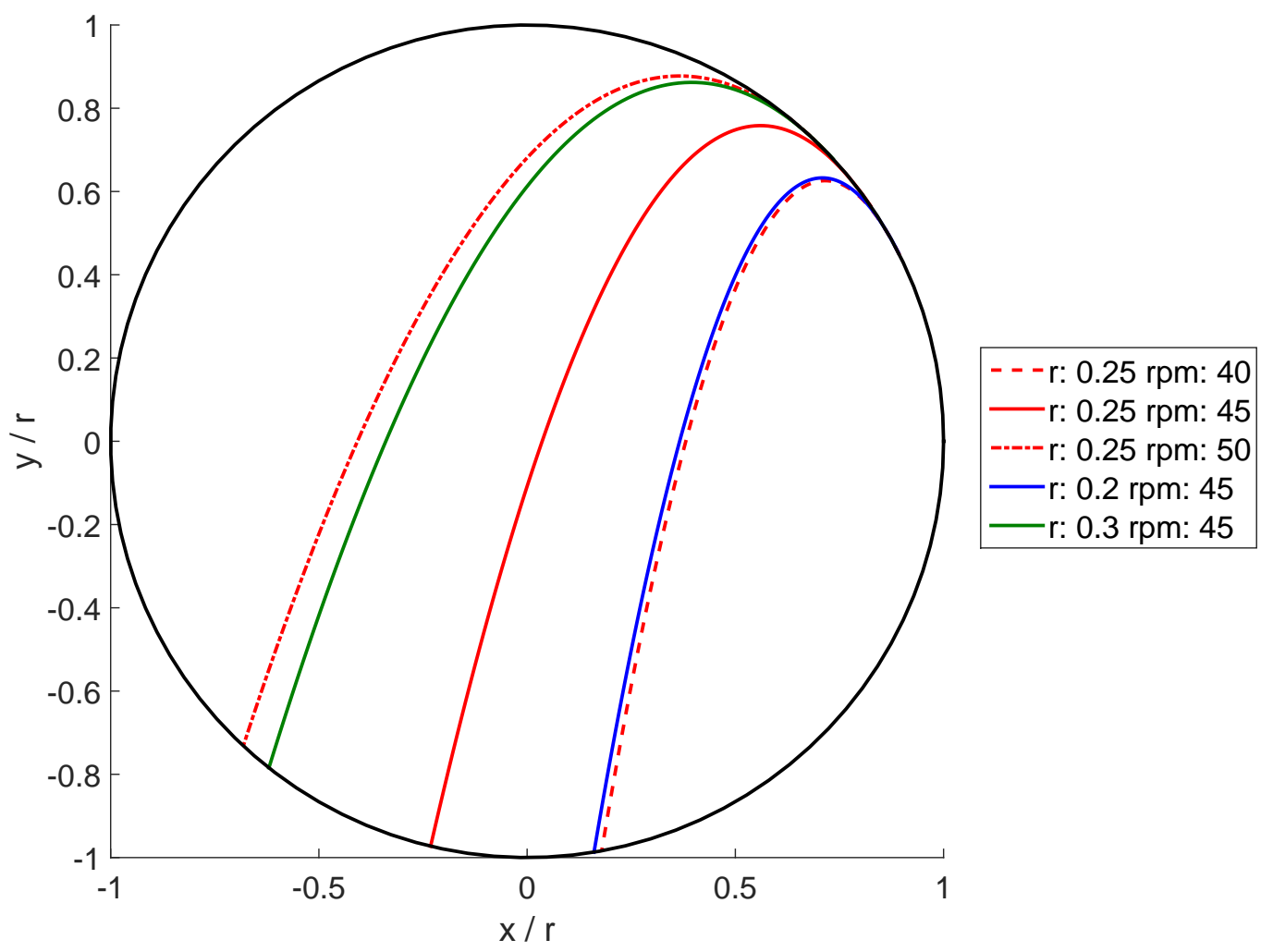

As expected from (3), the faster the rotational speed of the drum the higher vertically up the drum the clothing item will reach before detachment occurs. Similarly the larger the drum radius the higher up the side of the drum the detachment angle will be located.

At the point of detachment the initial velocity of the clothing mass is assumed to be tangential to the drum. The vanes within the drum may change the direction of the initial velocity and in turn change the flight time and trajectory 
Figure 3: Variation of point mass clothing trajectory with non-tangential departure velocity with drum speed of $45 \mathrm{rpm}$.

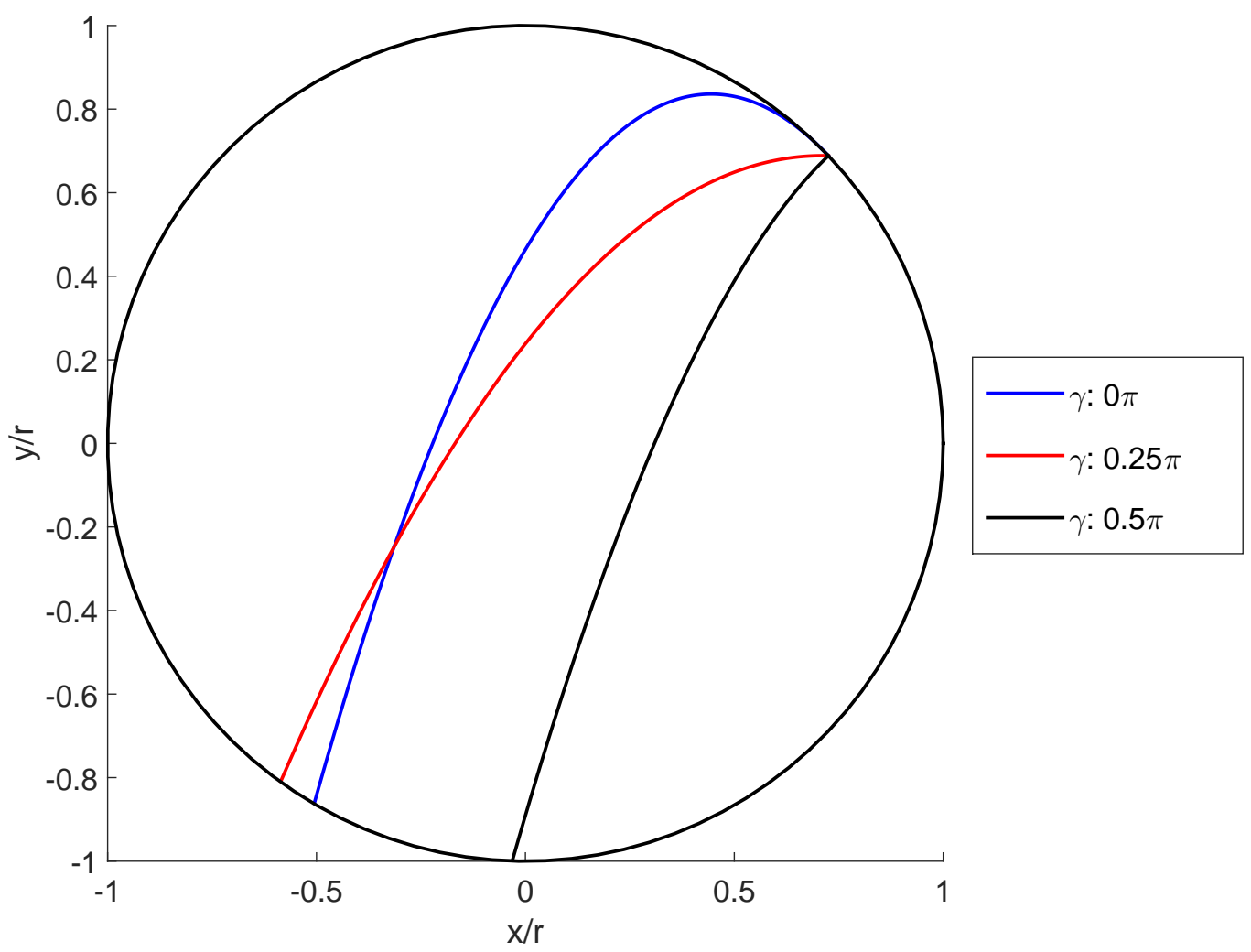

of the clothing mass. Figure 3 shows the trajectories when the direction of the initial velocity is rotated anticlockwise by some angle $\gamma$. While a $\gamma$ value of $0.5 \pi$ is not an expected initial velocity direction, it was chosen to show the range of possible trajectories for the clothing item rather than the expected trajectories. Varying the initial velocity at departure alters the clothing trajectory. Hence the time-of-flight and location of re-attachment at the base of the drum are also altered. The direction of the departure velocity as a result of vane shape and placement is of importance when optimising efficiency 
of the wash as a shorter time-of-flight may be achieved if the clothing leaves the drum edge with a different direction of detachment velocity. Therefore in order to model the trajectories and re-attachment location more accurately incorporating the effects of vanes on the detachment angle and velocity at detachment is important.

The trajectories predicted by the mechanistic model are similar in shape to those measured using positron emission particle tracking by Mac Namara et al. (2012). The mechanistic model under-predicts the departure angles obtained experimentally by Fisher \& Paykel Appliances and shown in Figure 9. The discrepancy is attributed to the use of a single clothing item in this model.

\subsection{Incorporating load size}

The above analysis considers a single item of clothing approximated by a point mass. This simplification, although helpful to enable analytic investigation, nonetheless assumes the particle has no structural features and its motion is not affected by other items of clothing in the machine drum. This is unrealistic and may contribute to the discrepancy in departure angle seen between this simple analytical model and the experimental data shown in Figure 9. However, this simple model provides a relationship between drum radius, drum rotational speed, departure and landing angles. The calculated trajectories are qualitatively similar to those reported by Mac Namara et al. (2012).

The single-item point-mass assumption is relaxed by assuming that when the drum is rotating the clothes occupy some fraction $p$ of the washing machine and have constant density in this fraction. At higher spin speeds clothes are flung further and have a reduced density, hence the value of $p$ increases with the speed of the drum. For simplicity we assume $p$ increases linearly with respect to $\omega$, that is $p=k_{1}+k_{2} \omega$, where $k_{1}, k_{2}>0$ are constants.

The cross-sectional area of the drum filled with clothes is then $A=p \pi r^{2}$.

The area that the clothes take up in the drum is bound by the clothing free 
fall trajectory in (4). This area determines the detachment angle $\theta_{\mathrm{d}}$ and the calculation of $\theta_{d}$ is an inverse problem that requires numerical methods. Specifically, the area under the free fall trajectory was computed using the trapezoidal rule, and a root finding method was used to determine the value of $\theta_{d}$ that gives the specified value of $A$.

For the values

$$
g=9.81 \mathrm{~m} \mathrm{~s}^{-2}, \quad r=0.248 \mathrm{~m}, \quad \mathrm{k}_{1}=0.4, \quad \mathrm{k}_{2}=0.1,
$$

the detachment angle and free fall paths are calculated for several different values of $\omega$, shown in Figure 4. A drum radius of $0.248 \mathrm{~m}$ was selected as this corresponded to one of the datasets provided by Fisher \& Paykel Appliances.

Figure 5 shows the results from the inverse problem over a range of values of $\omega$. The value of $E=\|\Delta v\| / T$, which represents the effectiveness of the washing machine, is maximised at approximately $\omega=\frac{36 \pi}{30}$ (i.e., $36 \mathrm{rpm}$ ).

\section{$3 \quad$ Numerical model of Discrete Elements}

A numerical model of the clothing motion in the drum was developed using the Discrete Element Method (DEM). The trajectories of articles of clothing around the washing machine were numerically modelled in LIGGGHTS open source soft-sphere DEM particle simulation software (Kloss et al. 2012). Deen et al. (2007) fully detailed the DEM in their comprehensive review. In brief, particle trajectories are tracked by numerical integration of the force and torque balances acting on each particle. For a particle, $i$, the force balance is

$$
m_{i} \frac{d v_{i}}{d t}=\sum_{j=1}^{N}\left(F_{n, i j}+F_{t, i j}\right)+m_{i} g
$$

where $v_{i}$ is the velocity of particle $i$, and $F_{n, i j}$ and $F_{t, i j}$ are the normal and tangential forces acting on particle $\boldsymbol{i}$ from contact with neighbouring particle $\boldsymbol{j}$. 
Figure 4: Free fall path for different drum speeds. $\theta_{\text {crit }}$ is shown as red triangles.
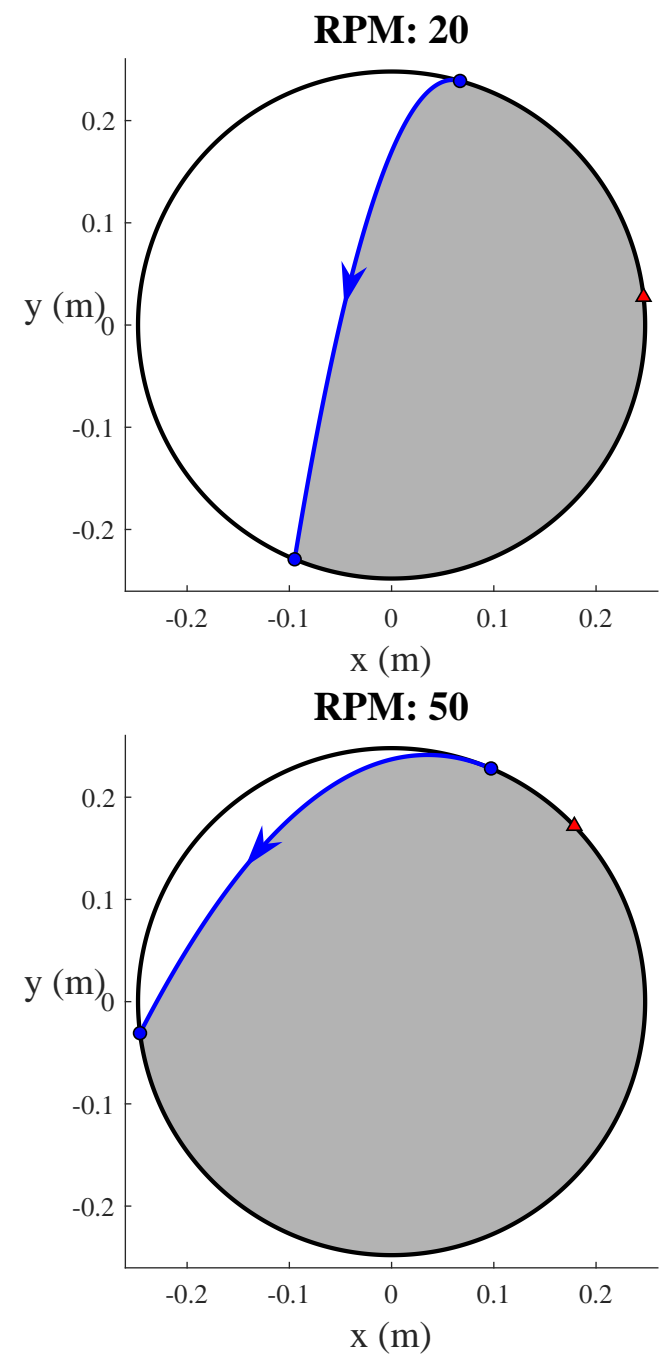

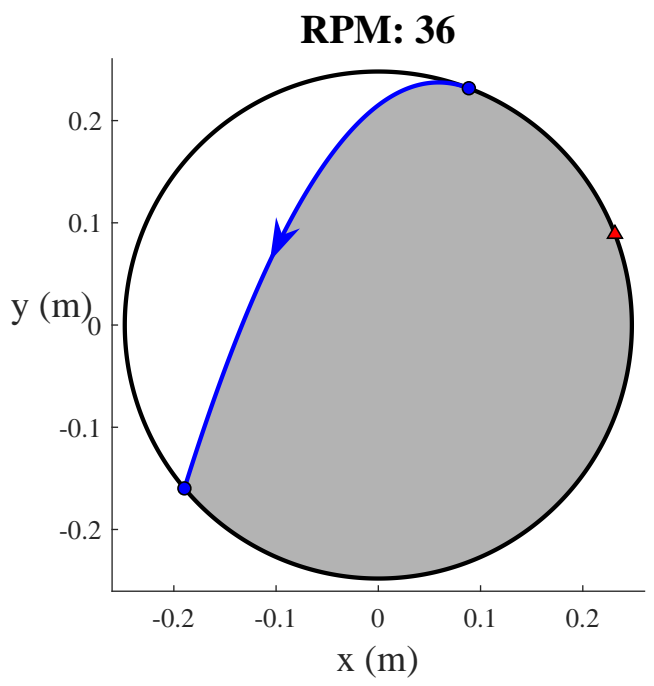


Figure 5: Kinematic relationships with drum speed

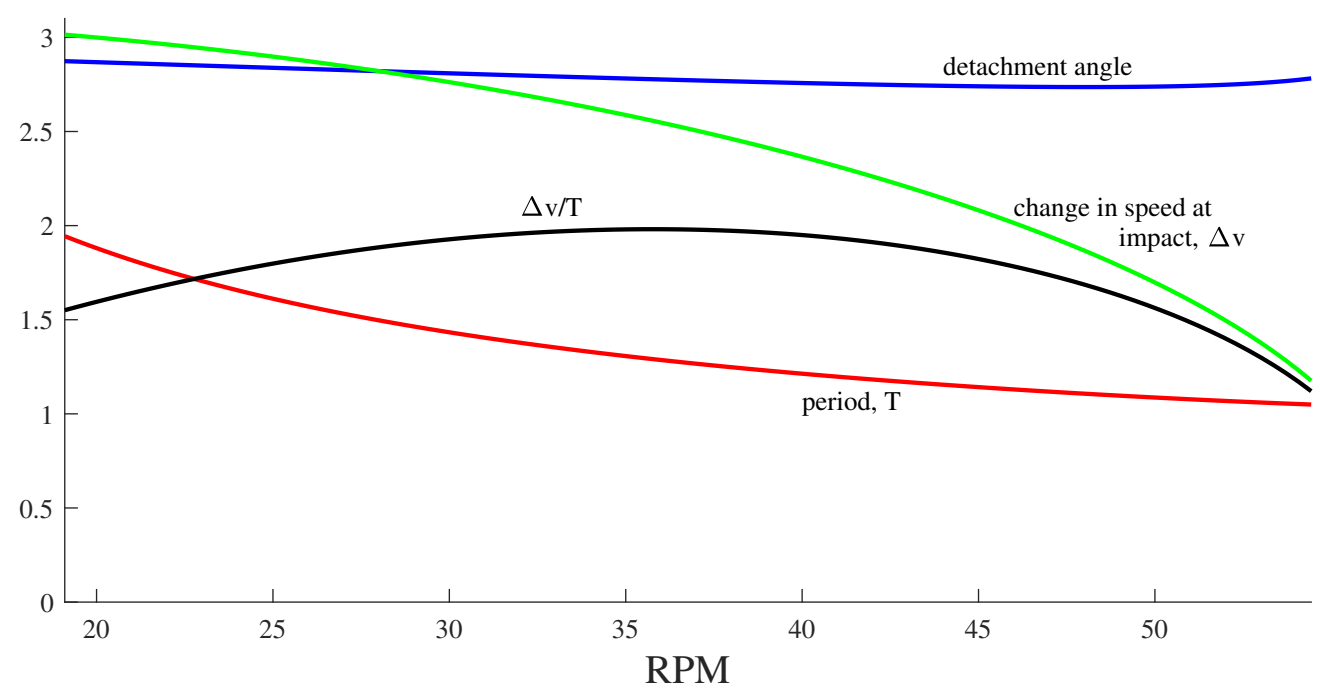

The torque balance on particle $i$ is calculated by

$$
I_{i} \frac{d \omega_{i}}{d t}=\sum_{j=1}^{N}\left(R n_{i j} \times F_{t, i j}-\frac{\mu_{r} R\left|F_{n, i j}\right| \omega_{t, i j}}{\left|\omega_{t, i j}\right|}\right),
$$

where $I_{i}$ is the moment of inertia for particle $i$. The particle radius is denoted by $R$. The coefficient of rolling friction is $\mu_{r}$ and $\omega_{t_{i}, j}$ is the tangential relative angular velocity between particles $\boldsymbol{i}$ and $\boldsymbol{j}$. Soft-sphere DEM resolves contact between particles, or between a particle and a solid suface with the Hertzian model (Maio and Renzo 2005). The contact force components are:

$$
\begin{aligned}
& F_{n, i j}=k_{n} \delta_{n} n_{i j}+\gamma_{n} v_{n, i j} ; \\
& F_{t, i j}= \begin{cases}k_{t} \delta_{t}+\gamma_{t} v_{t, i j} & \text { if } F_{t, i j} \leqslant \mu\left|F_{n, i j}\right|, \\
-\mu\left|F_{n, i j}\right| t_{i j} & \text { otherwise, }\end{cases}
\end{aligned}
$$


where the overlapping distance between particles $i$ and $j$ is $\delta_{n}$, and the normal relative velocity between particles $i$ and $\boldsymbol{j}$ is denoted by $v_{n, i j}$. The normal and tangential unit vectors are denoted by $n_{i j}$ and $t_{i j}$ respectively. The tangential displacement during the contact is denoted by $\delta_{t}$, and $\nu_{t, i j}$ is the tangential relative velocity. The coefficient of friction is represented by $\mu$. The normal and tangential spring constants are

$$
\begin{aligned}
& k_{n}=\frac{4}{3} E^{*} \sqrt{R^{*} \delta_{n}}, \\
& k_{t}=8 G^{*} \sqrt{R^{*} \delta_{n}},
\end{aligned}
$$

where $1 / E^{*}=\left(1-v_{i}\right) / E_{i}+\left(1-v_{j}\right) / E_{j}$, E is the elastic modulus and $v$ is the Poisson ratio, and $1 / R^{*}=1 / R_{i}+1 / R_{j}$. The effective shear modulus, $G^{*}$, is related to the elastic moduli of the particles by

$$
\frac{1}{G^{*}}=\frac{2\left(2-v_{i}\right)\left(1+v_{i}\right)}{E_{i}}+\frac{2\left(2-v_{j}\right)\left(1+v_{j}\right)}{E_{j}} .
$$

The normal and tangential damping coefficients are

$$
\begin{aligned}
& \gamma_{n}=-2 \sqrt{\frac{10 E^{*} m^{*}}{6}} \frac{\log e_{n}\left(R^{*} \delta_{n}\right)^{1 / 4}}{\sqrt{\log ^{2} e_{n}+\pi^{2}}}, \\
& \gamma_{t}=-2 \sqrt{\frac{40 G^{*} m^{*}}{6}} \frac{\log e_{n}\left(R^{*} \delta_{n}\right)^{1 / 4}}{\sqrt{\log ^{2} e_{n}+\pi^{2}}}
\end{aligned}
$$

where $e_{n}$ is the coefficient of restitution and the effective particle mass $m=$ $m_{i} m_{j} /\left(m_{i}+m_{j}\right)$. LIGGGHTS uses the velocity-Verlet method (Verlet 1967) to obtain the positions and the velocities of the spheres. The drum geometry was generated in SolidWorks CAD software to give a Stereolithography STL file format mesh imported into the simulation software. Multiple items of clothing were approximated as soft inelastic spheres in the drum. The elastic modulus was set to $10^{7} \mathrm{~Pa}$, the Poisson ratio was set to 0.33 . The restitution coefficient was set to 0.06 to inhibit rebounding of clothing items from collections (i.e., collisions were inelastic). The friction and rolling friction coefficients were 
Figure 6: (a) Snapshot of DEM simulation of front loading washing machine. (b) Frequency distribution of detachment angle. Simulation with 13 spheres: solid line, 25 spheres: dashed line, 39 spheres: dash-dot line.
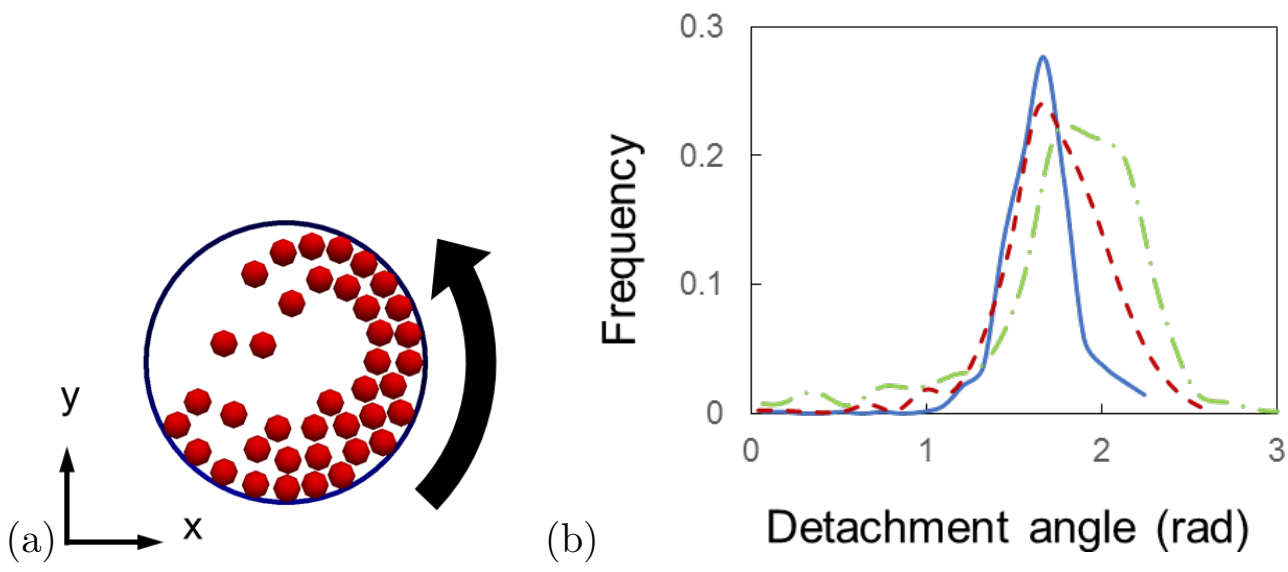

(b)

\section{Detachment angle (rad)}

set to arbitrary values of 100 to prevent spheres from rolling down the drum instead of being carried up and detaching. Three cases were modelled, where the drum was filled with 13, 25 and 39 spheres, each with a diameter of $0.05 \mathrm{~m}$ to approximate different sizes of washing load. The drum rotation was set to $45 \mathrm{rpm}$, and the simulations modelled $60 \mathrm{~s}$ of operation.

The departure angle was measured by recording the contacts between the particles and the drum over time. When a particle lost contact with the drum, its position was used to calculate the detachment angle. The frequency distribution of the departure angle is shown in Figure 6(b) and shows the mean clothing departure angle increases with load size. The leading article of clothing is pushed further up the drum by the following articles of clothing. The mean departure angle was slightly lower than the theoretical estimate from Equation (3) of $124^{\circ}(2.17 \mathrm{rad})$ from the bottom of the drum for $\omega=45 \mathrm{rpm}$, $r=0.248 \mathrm{~m}$, and $\mathrm{g}=9.81 \mathrm{~ms}^{-2}$. This discrepancy is attributed to slip between the spheres and the drum. If the spheres have a lower velocity than the drum, then the angle at which gravity exceeds the centripetal force is 
lower than if the spheres have the same velocity as the drum.

The discrete data was analysed by performing spatial averaging, where the particle information is allocated to spatial bins according to the position of the particle. The spatial bins in this study were equal in size to the particle radius, hence particles were simultaneously located in several bins. To accurately distribute particle information between bins occupied by particles, the correlation of Khawaja et al. (2012) was used. This correlation estimates the fraction of a particle in a spatial bin according to the overlap between the particle and the bin. Density and velocity fields of the spheres in the washing machine are shown in Figure 7 and shows regions of varying density and velocity within the drum. The spheres predominantly reside at the outer edge of the drum, as the wash load increases the spheres occupy the centre of the drum. The velocity maps are used to determine whether there are points where the clothes have a low velocity or are trapped, causing a poor wash performance. For example a stagnant zone in the centre of the drum is apparent for all wash loads. These maps are compared with the velocity fields produced by the image analysis (Section 5) or with velocity fields produced by other methods, such as radioactive tracking (Mac Namara et al. 2012).

\section{Data Analysis}

Fisher \& Paykel Appliances provided summaries of in-house testing, together with some original data sets, relating wash performance (SR and GA scores) to a number of investigated parameters. Furthermore, they shared their intuition, based on their studies, about the expected relationships between various parameters and the wash performance. One of the datasets provided by Fisher \& Paykel Appliances showed experimental results for SR and GA due to variations in the drum speed for a standard test rig. This experiment was duplicated for two fixed parameters, the time duration of the test cycle and the number of drum rotations within the cycle. A machine cycle is the time that a drum rotates in one direction before switching to the other direction. 
Figure 7: Space-time averaged volume fraction and velocity magnitude maps of DEM simulation data. The velocity vector field is overlaid on the velocity map to illustrate the trajectory of items of clothing at different positions in the washing machine.

\section{Spheres}
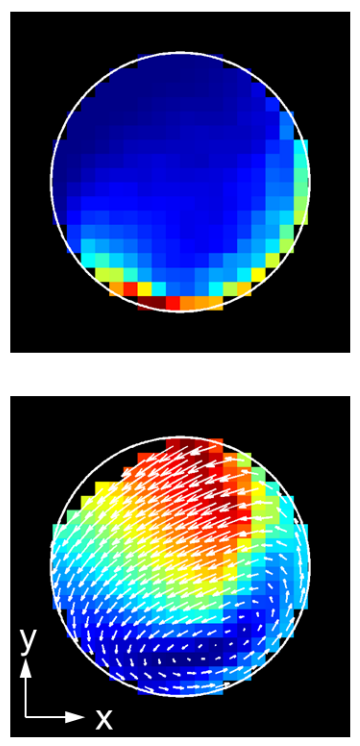

25 Spheres
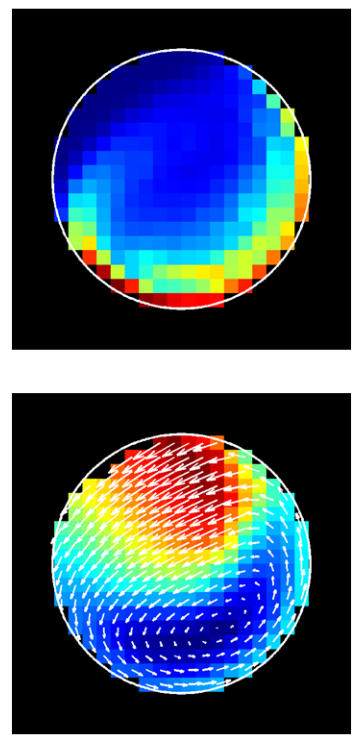

39 Spheres
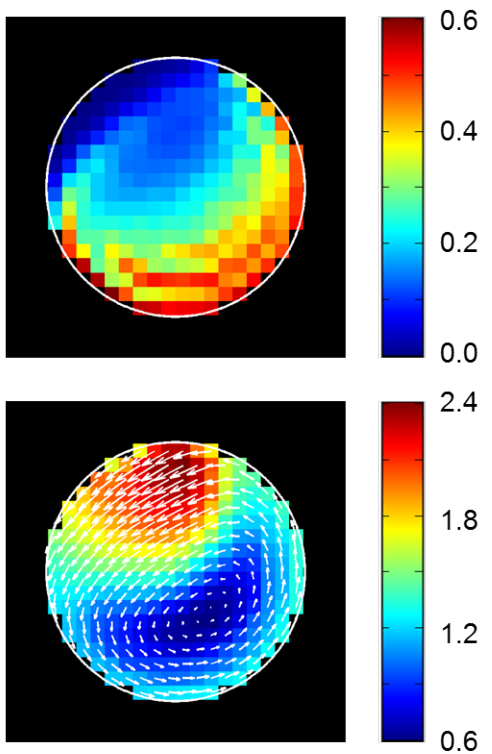

Figure 8 summarises this dataset. Analysis of this data indicates that:

- SR and GA are not correlated;

- landing location and departure location within the drum are correlated to the drum speed;

- SR varies with the drum speed, and hence landing angle; however, there is high variability within the test results at each speed;

- GA varies with the drum speed. 
Figure 8: Pairwise exploration of experimental results for soil removal and gentleness of action (inverse fray) from a dataset provided by Fisher \& Paykel Appliances. Drum speed [rpm], and cycle time are the independent variables, with landing and departure angles dependent variables.
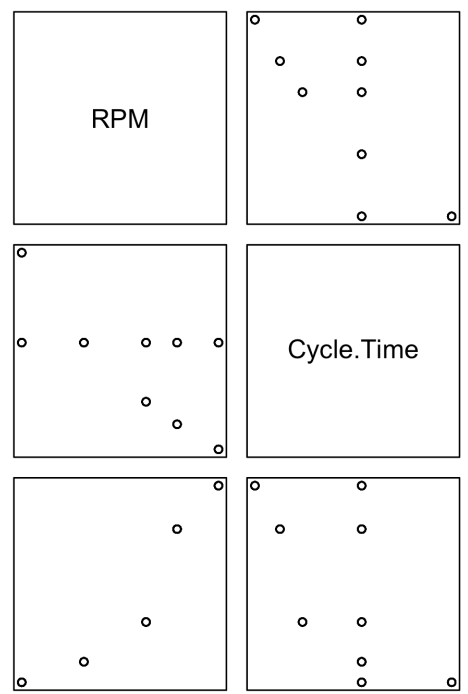

O
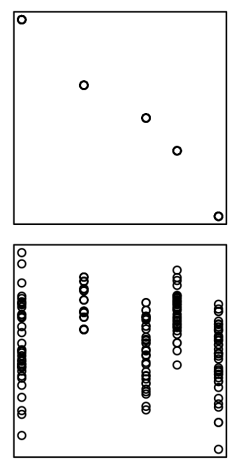

0
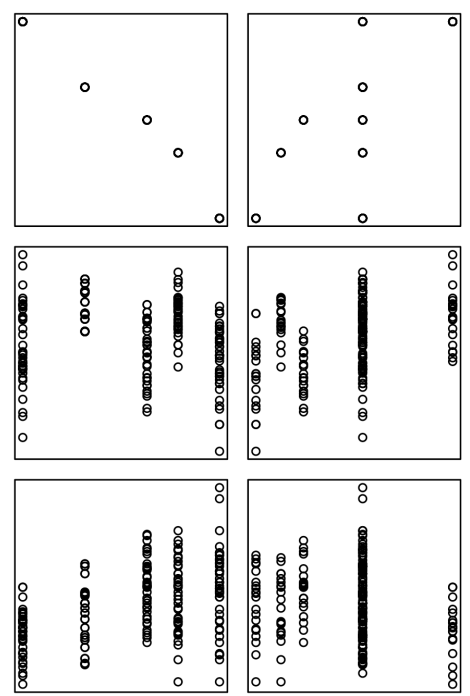
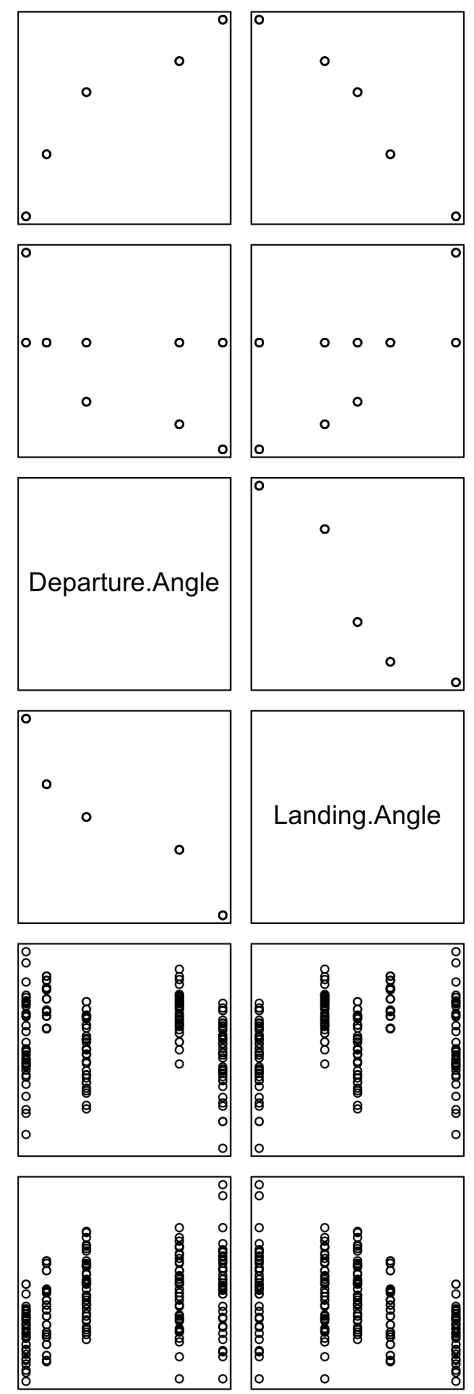
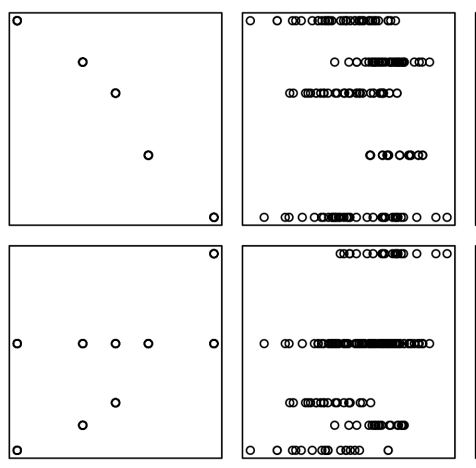

- 0 00000 0
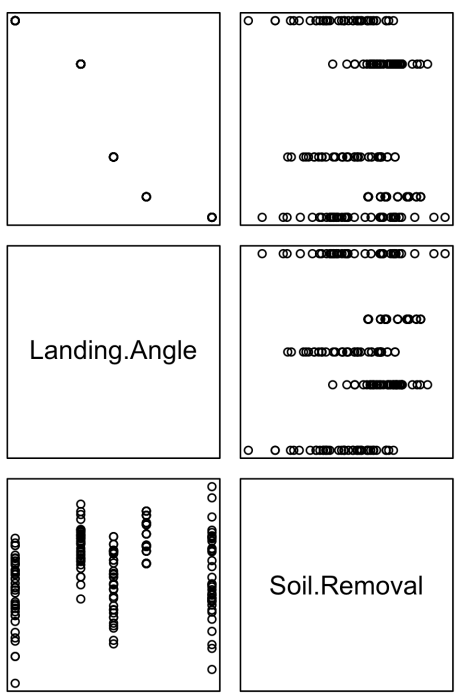

- 0 cocamparionos

Soil.Removal
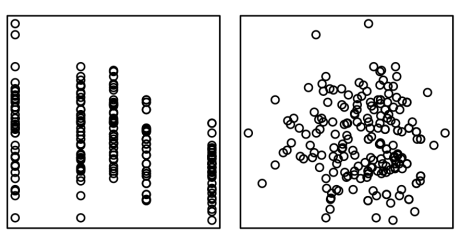
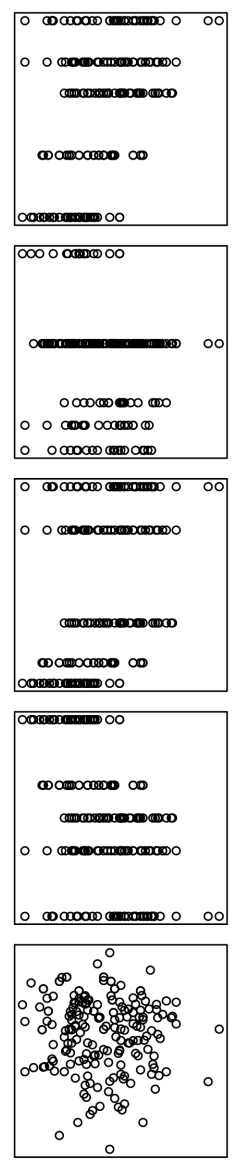

Fray 
It was somewhat unexpected that the SR and GA were not correlated. Actions that increase SR, such as garments rubbing against the drum and the impact with the base of the machine, could reasonably be expected to also increase wear and tear on the garment. This implies that other factors, held constant in this experiment, mitigate wear and tear without reducing SR. It is therefore feasible to improve soil removal without compromising gentleness of action.

Further investigation of this data failed to elicit a relationship that could be used to build a mathematical model of the wash performance. However, knowledge of washing mechanisms provides insight into the physical features of a wash that increase the performance. The action of dropping the clothing to the base of the drum is known to facilitate soil removal. In a front-loading washing machine, vanes fixed to the side of the drum pick up clothing as the drum rotates. At low spin speeds, when the centripetal force is less than the gravitational force, the clothing is carried around the side of the drum before falling to the base of the drum to be picked up by a subsequent vane. The point on the drum where the clothing leaves the side of the drum forms the departure angle, while the point of the drum where the clothing lands forms the landing angle. Wash efficiency is improved by minimising the time garments roll along the base of the drum before being lifted by the next vane. This rolling action increases wear and tear on garments whilst having a negligible effect on soil removal. The action of landing at the base of the drum is a significant component of soil removal. Therefore, increasing the number of 'flips' that a garment does is expected to increase soil removal.

To investigate the impact of vane placement on wash performance a datadriven model of landing and departure angles as a function of drum speed $(\Omega)$ is developed. Insight from the kinematic model, proposed in Section 2, indicates a quadratic relationship between landing or departure angle and drum speed is appropriate. The linear regression model $l m$ (R Core Team 2017 ) is used to fit the data to a quadratic model, resulting in the relationships

$$
\begin{aligned}
& \theta_{d}=150.29-2.225 \Omega+0.048 \Omega^{2}, \\
& \theta_{l}=42.939+0.6228 \Omega-0.0549 \Omega^{2},
\end{aligned}
$$


where $\Omega[\mathrm{rpm}]$ is the angular speed of the drum, $\theta_{\mathrm{d}}\left[^{\circ}\right]$ is the location at which garments depart the drum, and $\theta_{l}\left[{ }^{\circ}\right]$ is the landing location of garments. For dimensional consistency the coefficients have units $\left[^{\circ}\right]$ for the constant term, $\left[{ }^{\circ} / \mathrm{rpm}\right]$ for the linear term, and $\left[{ }^{\circ} / \mathrm{rpm}^{2}\right]$ for the quadratic term. The drum is assumed to be rotating anticlockwise. Figure 9 shows the proposed model together with the experimental data used to train the model. Angles are measured anticlockwise from the base as shown in Figure 10.

Garments are collected by a vane at the base of the drum, travelling on the vane until reaching the departure angle $\theta_{\mathrm{d}}$, see Figure 10. At this point, the garments roll off the vane and drop to the base of the drum, at location $\theta_{l}$. To maximise soil removal and gentleness of action, ideally garments have as many 'flips' (or landings) as possible, with minimal roll at the bottom of the drum. This is achieved by matching the drum speed and vane location. This analysis, based on data provided by Fisher \& Paykel Appliances, considers their 'standard' mixed load. This means that the load is designed to mimic typical usage by householders, and as such clothing may land either on the drum or on other items of clothing. Testing would be required to determine whether the relationships observed in previous experiments would continue to hold with changes in vane placement.

There are three distinct components of motion to consider for garments when seeking to maximise 'flips' and reduce roll at the bottom of the drum. First the garments are collected by a vane, travel on the vane around the edge of the drum until reaching the departure angle $\theta_{d}$. Second, the garment falls off the vane and fall freely towards the base of the drum, landing at the location $\theta_{l}$. During this time, the drum continues to rotate. The rotation of the drum during this time period is given by the time taken for clothing to fall to the base of the drum and the rotational speed of the drum, that is $\theta_{\text {fall }}=\mathrm{t}_{\text {flight }} \Omega / 60$ (as the flight time has units seconds). Using the flight time given by the kinematic model (see (5) and (3)), the angle through which the 
Figure 9: Comparison of the quadratic models for departure and landing angles with the experimental data. The quadratic fits return adjusted Rsquared values of 0.955 and 0.975 respectively. The F-statistics $\gg 1$ (202 and 369 respectively), which supports the use of these models to predict departure and landing angles as a function of drum speed.
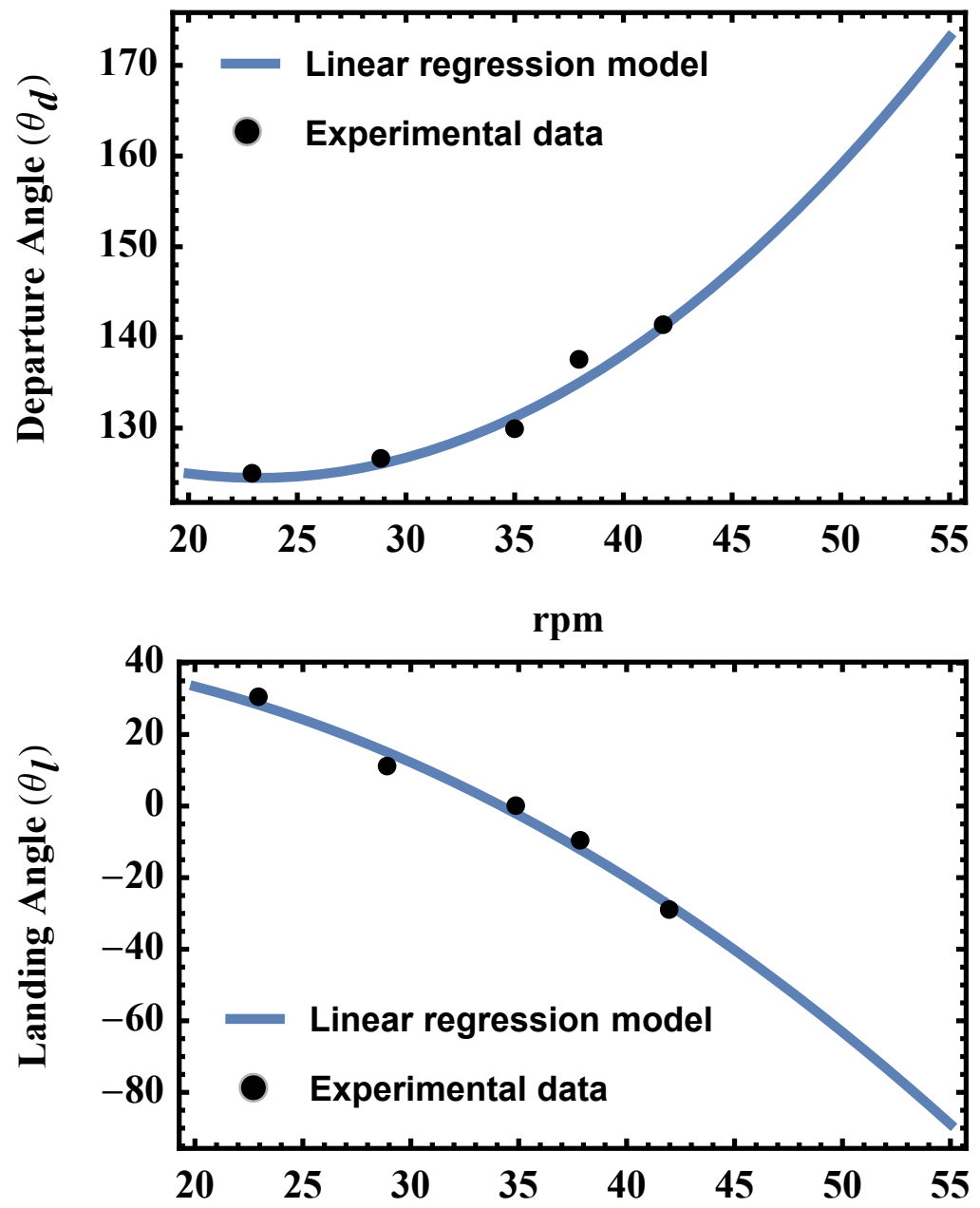

rpm 
Figure 10: Schematic of a garment in a front loading washing machine with three-vane configuration. $\phi$ is the angle between the garment's landing location and the vane that next picks up the garment.
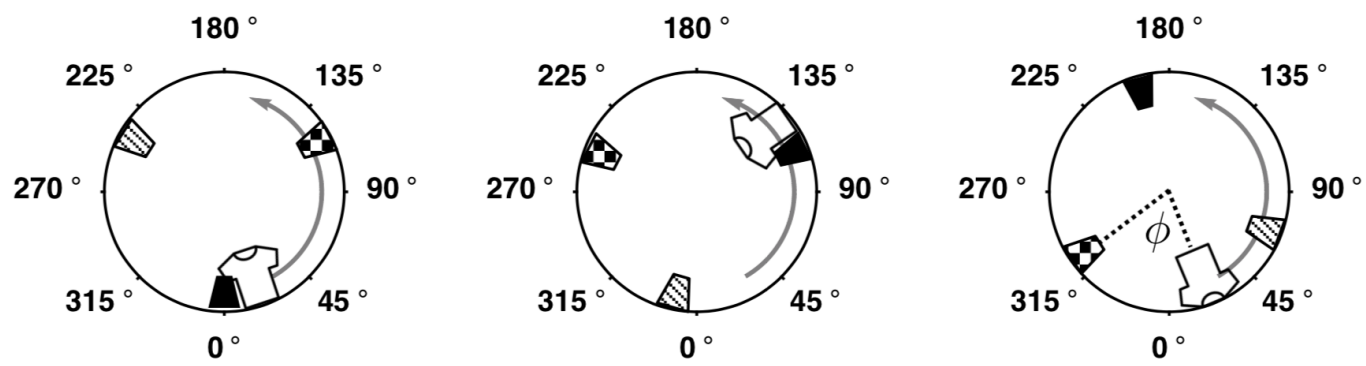

drum rotates while the garment is falling is

$$
\theta_{\text {fall }}(\Omega, r)=\frac{24 r \omega \Omega}{g} \sqrt{1-\frac{r^{2} \omega^{4}}{g^{2}}} .
$$

The third phase consists of the garment rolling along the base of the drum until it is collected by the next vane. Ideally, this rolling phase is minimised. The angular rotation of the drum from when a garment is picked up by the first vane until it lands on the base of the drum (at $\left.\theta_{l}\right)$ is $\theta_{d}+\theta_{\text {fall }}$. Therefore, at time $t=0$ (when the garments are first picked up from the base of the drum) the ideal location of the 'next' vane is

$$
\Phi=\theta_{\mathrm{l}}(\Omega)-\left[\theta_{\mathrm{d}}(\Omega)+\theta_{\text {fall }}(\Omega, \mathrm{r})\right]-\epsilon \quad\left(\operatorname{modulo} 360^{\circ}\right),
$$

where $\epsilon$ is some small angle to ensure that the clothing falls in front of the vane. The 'first' vane is assumed to be located at $\theta=0$ at the point of collection, the natural resting point of clothing within the drum.

Typically, front-loading washing machines have been developed with a threevane configuration, such as in Figure 10. That is, with vanes located at $\theta=0^{\circ}, 120^{\circ}$, and $240^{\circ}$. Figure 11 explores the landing angle relative to the 
location of the next vane for different vane configurations and drum speeds. To maximise the number of flips while reducing the roll along the bottom, the difference, $\phi$, between landing angle and the location of the next vane should be as close to zero as practicable. Fisher \& Paykel Appliances can use this approach to identify the optimal drum speeds for different vane configurations, or alternatively the optimal vane configure for a specified drum speed, at least as it pertains to maximising 'flips' while reducing roll. The above analysis indicates that, for certain drum speeds, a four-vane configuration could provide improved soil removal over the standard three-vane configuration. At $40 \mathrm{rpm}$ (which falls within the typical range of drum speeds for front loading washing machines), our analysis indicates that a four-vane configuration may be superior to not only the standard three-vane but also the five and six-vane configurations explored as this minimises the angle through which clothes would roll before being collected by the next vane. As different wash programs operate at different drum speeds (and even within a single program drum speed can vary), the range of drum speeds applicable to a machine must be considered. Nevertheless, this analysis provides a way to help identify optimal drum speeds for different vane configurations. For example, with a three-vane configuration, our results suggest that the optimal drum speed to maximise 'flips' while reducing roll would be approximately $37 \mathrm{rpm}$, while for a five-vane configuration (which may have practical limitations given the size of drums) optimal speeds are evident at approximately 24, 34 and $43 \mathrm{rpm}$. These theoretical results require practical testing to determine how well they translate from the idealised model to a mixed load of clothing. However, they provide novel insight into a potential alternative way to achieve improved washing performance. Furthermore, consideration of the full range of drum speeds across all wash cycles available in a machine would be required before undertaking such a change to the configuration of the drum. 
Figure 11: Exploration of the angle $\phi$ between a garment at landing and the location of the next vane for different drum speeds and vane configurations.

3-vane configuration

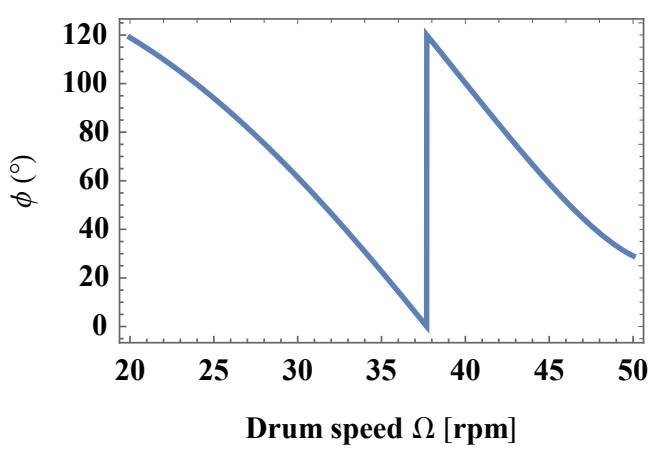

5 -vane configuration

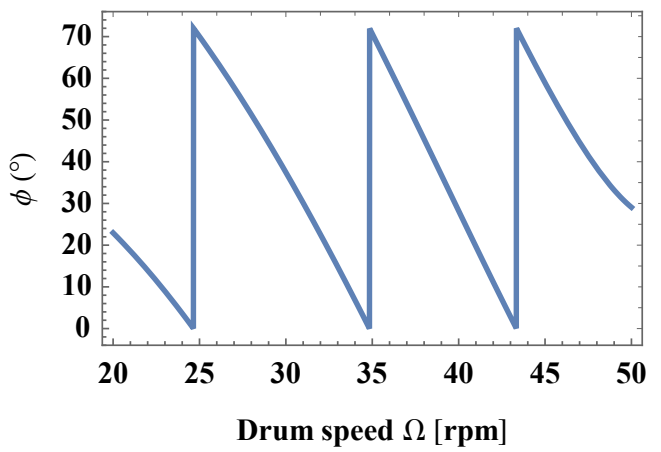

4-vane configuration

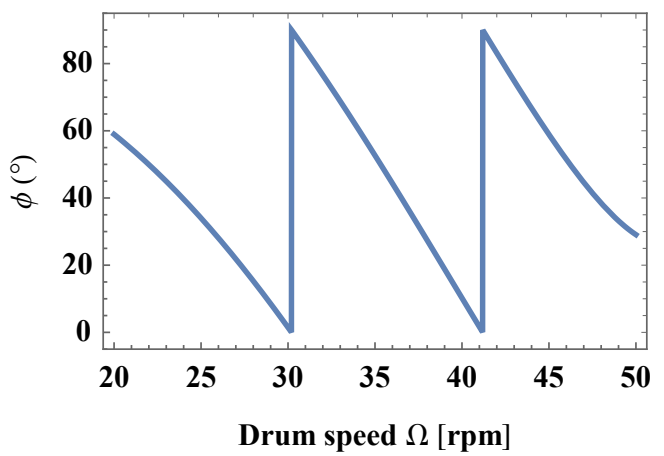

6-vane configuration

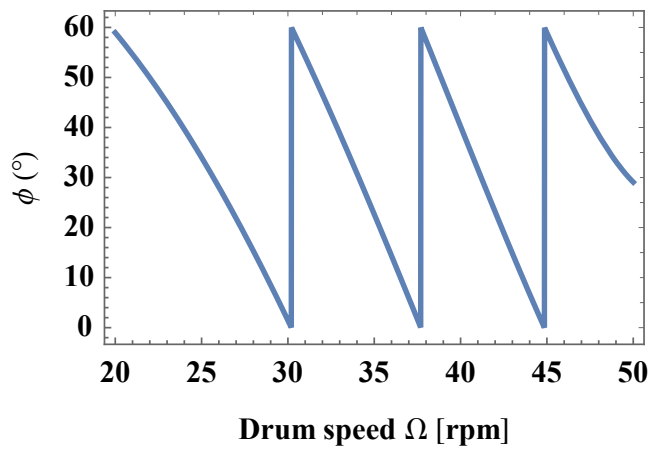




\section{Empirical Clothing Motion}

The analytical model described in Section 2 models a garment in the drum as a point mass, and accounts for support of the remaining clothes in the drum by matching the area under the point mass' free-fall path to a given fraction of the drum area. The discrete element method described in Section 3 models clothing as non-overlapping inelastic spheres. As these methods do not fully account for the overlapping and mixed nature of a clothing load spinning in a drum, a method for empirically validating the analytical and discrete element models using video analysis of the motion of clothing in the drum was developed. Fisher \& Paykel Appliances provided example videos of front loading washing machine operation as background information.

In this section, a software system for continuously recording the position, shape, and velocity of clothing items in the front loader drum as seen through the glass door is described. These measurements of position and velocity were taken from a region near the vertical plane at the front of the drum, visible to the camera, and not obscured from view by clothing. While measurements further to the rear of the drum were not obtainable, the front plane is expected to be representative.

The video feed is analysed frame by frame. As the video is unstabilised, the first step is to detect the circular perimeter of the glass door of the front loading machine, and use this as a point of reference to stabilise and scale the video. The SURF method (Bay et al. 2008) was used to locate key points in each frame. An approximate nearest neighbours method matched key points between each frame and the following frame. Using hundreds of key points per frame, the change in position of each local region of visible clothing items was determined. To eliminate false matches, several methods for filtering approximate feature matches were applied, using bounds on the expected change in position.

Measuring the change in position of key points between frames, corresponding to local areas of visible clothing, gives instantaneous velocities of each point. 
These were combined to derive vector fields for velocity, acceleration, and shear across the visible depth of clothing. These vector fields and derived information can be used to validate or improve the analytical and discrete element models, or used for direct evaluation of different washing cycles and drum configurations, and can be related to wash performance, both cleaning and fray.

The obtained vector fields were smoothed using several methods. The simplest method used was applying a standard grid to each frame, and averaging the velocities obtained for key points in each grid cell. Other methods include fitting a spline surface. Figure 12 shows averaged values of the velocity field for two different loads, one half full, and one near full. These figures display average velocity magnitude for each grid cell, averaged over 60 seconds. Figure $12 \mathrm{~b}$ displays a velocity field from an $8.5 \mathrm{~kg}$ load, with a nearly full drum. The clothing can be seen tracing a more circular path. The left velocity field, Figure 12a, was measured from a sub-full $5 \mathrm{~kg}$ load tracing a lumpier path towards the lower right area of the drum.

Figure 13 illustrates indicative points tracked between frames (blue lines), and grid bins (red squares). Other ways of visualising and storing the information are possible depending on use case. For example, a sliding window of $n$ seconds can be analysed, looking at back-and-forth cycles and the change in velocity over a short time.

Software for tracking the position and outline of an individual garment mixed in with a full load of other clothing was developed. The motion of this individual garment was tracked in the visible region of the drum, and the motion and position relative to other clothing items determined. This indicates the degree of mixing between clothes, and may also be useful information to correlate with wash performance and other machine and wash cycle parameters.

These videos were taken using a hand-held phone camera, with a frame rate of $30 \mathrm{fps}$ and imperfect lighting conditions, including inadequate lighting and obscuring reflections. Video capture designed with this process in mind, taken 
Figure 12: Averaged velocity field magnitudes for clothing rotating in the drum

(a) A half-full load, with a clear (b) A full load showing more circular parabolic fall trajectory motion
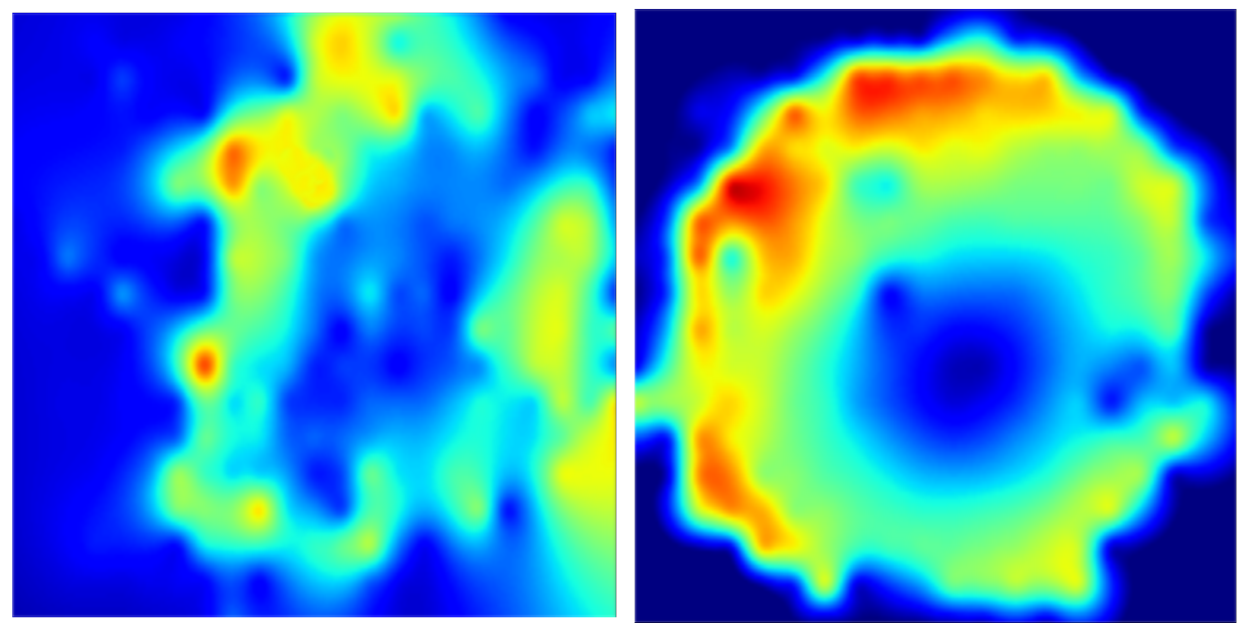

with a tripod-mounted camera, a $120 \mathrm{fps}$ frame rate, and better lighting would significantly improve the results presented here.

Another method for direct measurement of clothing motion in a front or top loading machine is Position Emission Particle Tracking (PEPT). However, this process involves expensive and specialised equipment (Mac Namara et al. 2012). Analysing video of clothing motion in the drum is a much less expensive proxy. 
Figure 13: Composite image showing the velocity averaging bins, the velocity field magnitude heat-map, and the motion of several hundred key-points between two frames.

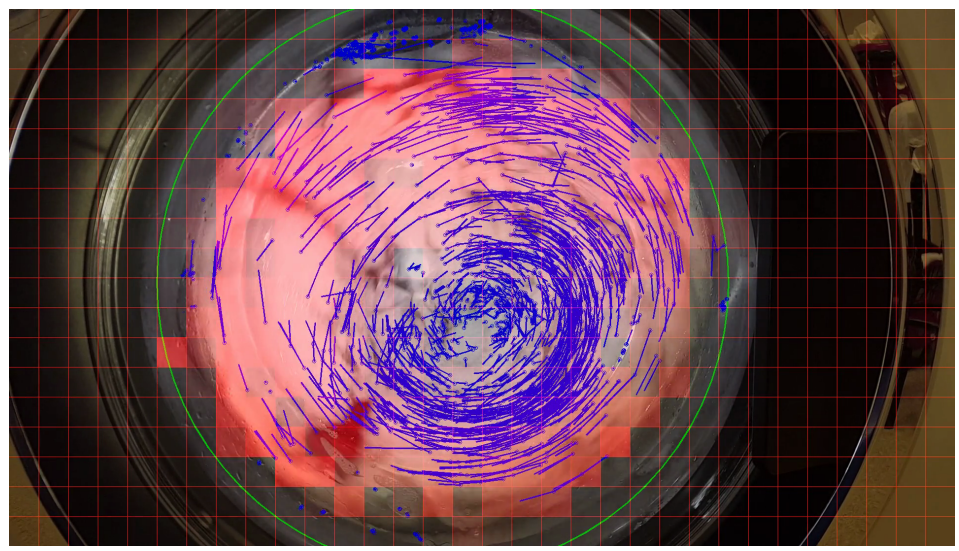

\section{Summary}

The impact of variation in the mechanical characteristics of a front-loading washing machine on wash parameters has been investigated using a combination of data analytics, physical modelling, and video analysis. These investigations demonstrate that further analysis and modelling would benefit the design and product evaluation processes for Fisher \& Paykel Appliances.

Analysis of the data did not elicit a relationship between gentleness of action and soil removal, indicating that improved wash performance across both criteria simultaneously is likely possible. A hypothesised four-vane configuration, as opposed to the traditional three-vanes currently in use, has the potential to provide an improved wash performance. The four-vane configuration has two different drum speeds that still cause the clothing to fall at the desired location directly in front of the next vane, minimising the roll along the base of the drum (improved gentleness of action) while maximising over a cycle 
the number of times clothes fall (improved soil removal).

Physical models of the motion of a point mass clothing item within the washing machine drum showed kinematic relationships between drum speed and clothing departure angle, change in clothing velocity on landing, and period of the motion. Sensitivity analysis of the trajectory of a point mass clothing item to drum speed and radius showed the faster the rotational speed of the drum, the higher up the drum the clothing item reaches before detachment occurs. Similarly, the larger the drum radius, the higher up the side of the drum the detachment angle is located. Numerical modelling using Discrete Elements of the trajectories of multiple items of clothing approximated as soft inelastic spheres in the drum showed mean clothing departure angle increased with load size as the leading article of clothing is pushed further up the drum by the following articles of clothing. Velocity fields of the clothing spheres in the drum generated from the models can be used to determine whether there are points where the clothes have a low velocity or are trapped causing a poor wash performance.

The developed software for tracking the position and outline of individual garments provides a novel mechanism to understand the mixing of clothing within a wash, and provides a means to improve data collection for Fisher \& Paykel Appliances. Further exploration of the opportunities provided through video analysis are recommended.

\section{Acknowledgements}

We are grateful to the industry representatives Kirsty Davies and Jennifer Trittschuh for their active contributions to this work. We also thank the many people who contributed to this project: Daniel Corrigan, Gareth Gordon, Hyoungsuk So, Hyuck Chung, Irvin Chew, Liam Bignell, Mathieu Sellier, Wenjun Zhang and Xin Zhou. 


\section{References}

Bay, Herbert et al. (June 2008). "Speeded-Up Robust Features (SURF)". In: Computer Vision and Image Understanding 110.3, pp. 346-359. ISSN: 1077-3142. DOI: 10.1016/j.cviu.2007.09.014 (cit. on p. M55).

Deen, N.G. et al. (2007). "Review of discrete particle modeling of fluidized beds". In: Chemical Engineering Science 62.1. Fluidized Bed Applications, pp. 28-44. ISSN: 0009-2509. DOI: $10.1016 / \mathrm{j}$. ces.2006.08.014 (cit. on p. M41).

Fullard, Luke et al. (Dec. 2016). "Eliminating early cut-offs and estimating cycle end time in a tumble dryer". In: Proceedings of the 2015 Mathematics and Statistics in Industry NZ Study Group, MISG-2015.

Ed. by Graeme Wake and A. J. Roberts. Vol. 57. ANZIAM J. Pp. M237-M267. DOI: 10.21914/anziamj.v57i0.10523 (cit. on p. M33).

Khawaja, H. et al. (June 2012). "Quantitative analysis of accuracy of voidage computations in CFD-DEM simulations". In: Journal of Computational Multiphase Flows 4 (2), pp. 183-192 (cit. on p. M46).

Kloss, Christoph et al. (2012). "Models, algorithms and validation for opensource DEM and CFD-DEM". In: Progress in Computational Fluid Dynamics, an International Journal 12.2-3, pp. 140-152. DOI: 10.1504/PCFD. 2012.047457 (cit. on p. M41).

Lee, Jung-Hyo et al. (2007). "Load modeling for the drum washing machine system simulation". In: 2007 7th Internatonal Conference on Power Electronics, pp. 861-865. DOI: 10.1109/ICPE.2007.4692508 (cit. on p. M35).

Mac Namara, C. et al. (June 2012). "Dynamics of textile motion in a front-loading domestic washing machine". In: Chemical Engineering Science 75, pp. 14-27 (cit. on pp. M40, M46, M57).

Maio, F. P. Di and A. Di Renzo (2005). "Modelling Particle Contacts in Distinct Element Simulations: Linear and Non-Linear Approach". In: 
Chemical Engineering Research and Design 83.11, pp. 1287-1297. ISSN: 0263-8762. DOI: 10.1205/cherd.05089 (cit. on p. M43).

Marsh, C. and A. Wilkins (2005). "Determining temperature control of wash water in a laundry environment". In: Proceedings of the 2005 Mathematics-in-Industry NZ Study Group, MISG-2015, pp. 163-208 (cit. on p. M33).

Marsh, Clive et al. (2005). "Developing an understanding of washing machine dynamics". In: Proceedings of the 2006 Mathematics-in-Industry Study Group, MISG-2015, pp. 133-152 (cit. on p. M33).

R Core Team (2017). R: A Language and Environment for Statistical Computing. R Foundation for Statistical Computing. Vienna, Austria. URL: https://www.R-project.org/ (cit. on p. M49).

Verlet, Loup (1967). "Computer "Experiments" on Classical Fluids. I. Thermodynamical Properties of Lennard-Jones Molecules". In: Physical Review 159 (1), pp. 98-103. DOI: 10.1103/PhysRev.159.98 (cit. on p. M44).

Yun, Changsang and Chung Hee Park (2015). "The effect of fabric movement on washing performance in a front-loading washer II: under various physical washing conditions". In: Textile Research Journal 85.3, pp. 251-261. DOI: 10.1177/0040517514545260 (cit. on p. M35).

\section{Author addresses}

1. Melanie E. Roberts, IBM Research Australia \& Australian Rivers Institute, Griffith University, Australia mailto:m.roberts20griffith.edu.au orcid:0000-0003-4027-9651

2. Celia Kueh, School of Engineering and Advanced Technology, Massey University, Palmerston North, New Zealand mailto:c.s.1.kueh@massey.ac.nz orcid:0000-0002-3505-803X 
3. Emma Greenbank, Victoria University of Wellington, New Zealand mailto: emma.greenbank@sms . vuw.ac.nz orcid:0000-0002-2332-3844

4. Daniel Clarke, University of Canterbury, Christchurch, New Zealand mailto:dac136@uclive.ac.nz orcid:0000-0001-7668-2472

5. Sibylle van Hove, University of Auckland, New Zealand mailto:svan511@aucklanduni.ac.nz orcid:0000-0003-0789-2665

6. David J.W. Simpson, Institute of Fundamental Sciences, Massey University, Palmerston North, New Zealand

mailto:d.j.w.simpson@massey.ac.nz orcid:0000-0002-0284-6283

7. Alan Williams, Isogonal, New Zealand mailto:alan@isogonal.co.nz

8. James Williams, Isogonal, New Zealand mailto: james@isogonal.co.nz 